\title{
Transportation behaviour of radioactive substances in soils
}

Shinya Inazumi PhD

Associate Professor, Department of Civil Engineering, Shibaura Institute of

Technology, Tokyo, Japan (corresponding author: inazumi@shibaura-

it.ac.jp)

\author{
Hiroyuki Hashida \\ Executive Officer, Sumice Kenzai Co., Ltd, Tokyo, Japan \\ Yoji Ueyama \\ Graduate student, Department of Social Informatics, Kyoto University, \\ Kyoto, Japan
}

In 2011, a number of radioactive substances were released as a result of the accident at the Fukushima Daiichi Nuclear Power Plant, Japan, which was caused by the Tohoku region Pacific coast earthquake. The radioactive substances that were released fell onto the surrounding ground and into the sea. Therefore, decontamination measures for the ground have been conducted at the field site. However, the safety and validity of modern decontamination measures were uncertain because no analytical verification had been performed that considered the various properties of the ground. Moreover, selection of the site where storage containers for radioactive materials could be constructed was a critical issue that required a solution. Therefore, it was necessary to evaluate quantitatively the behaviour of radioactive substances in soils to prepare new decontamination methods for the ground. In this study, the advection-dispersion equation was added to the radioactive half-life to evaluate the transportation of radioactive substances. As the results of this study show, this analytical method could recreate the on-site situation through comparison of the analysis results with the measurement results. Furthermore, modern decontamination techniques were effective for the section under analysis for ground with a silt or a clay layer. However, these techniques were not effective for sand layers.

\section{Notation}

C concentration of the radioactive substance

$C_{0} \quad$ initial concentration of the radioactive substance

$D^{*} \quad$ coefficient of molecular diffusion of the radioactive substance

$\boldsymbol{D}_{i j} \quad$ dispersion tensor

H total head

$K_{\mathrm{d}} \quad$ distribution coefficient

$k_{x} \quad$ hydraulic conductivity in the $x$ direction

$k_{y} \quad$ hydraulic conductivity in the $y$ direction

Q applied boundary flux

$S$ adsorption

$T_{1 / 2} \quad$ radioactive half-life

$t$ time

$U_{i} \quad$ Darcian velocity

$\|V\| \quad$ norm of actual flow

$x_{i} \quad i$ th $x$ coordinate

$x_{j} \quad j$ th $x$ coordinate

$x_{n} \quad$ distance of contaminant diffusion by water velocity when time $t$ passes

longitudinal dispersivity

transverse dispersivity

Kronecker delta

volumetric water content

decay coefficient

dry density of the soil

tortuosity

$i$ th seepage velocity

$j$ th seepage velocity

\section{Introduction}

The accident at the Fukushima Daiichi Nuclear Power Plant, Japan, was caused by the Tohoku region Pacific coast earthquake in 2011. Because of this accident, radioactive substances were released, carried by the wind and then fell on the ground and into the sea with subsequent rainfall. Iodine-131, caesium-134 and caesium-137 have been detected over a widespread area, including farmlands and the area surrounding the Tohoku and Kanto regions. It has been reported that approximately $22 \%$ of the released radioactive caesium fell to the ground (Morino et al., 2011). Generally, it was found that most of the radioactive substances deposited on the ground were present within $5 \mathrm{~cm}$ of the surface (Endo et al., 2012; Kato et al., 2012; Ministry of Education, Science and Culture, 2011a). Within the clay layer, the caesium existed within $2 \mathrm{~cm}$ of the surface (Shiozawa et al., 2011) because it is readily absorbed by clay particles.

The range of geoenvironmental pollution attributable to this accident was so massive that decontamination measures remain in progress. Generally, the decontamination measures for the soil include dredging and removal of almost $5 \mathrm{~cm}$ of the top soil layer (Ministry of the Environment, 2013). However, verification analysis considering the various soil properties has not been performed. Hence, the safety and validity of modern decontamination measures are thought to be uncertain. Moreover, selection of the site for constructing the storage containers used for radioactive materials is a critical issue that must be resolved. Therefore, it is necessary to evaluate quantitatively the behaviour of radioactive substances in the soil in order to prepare new decontamination measures. 


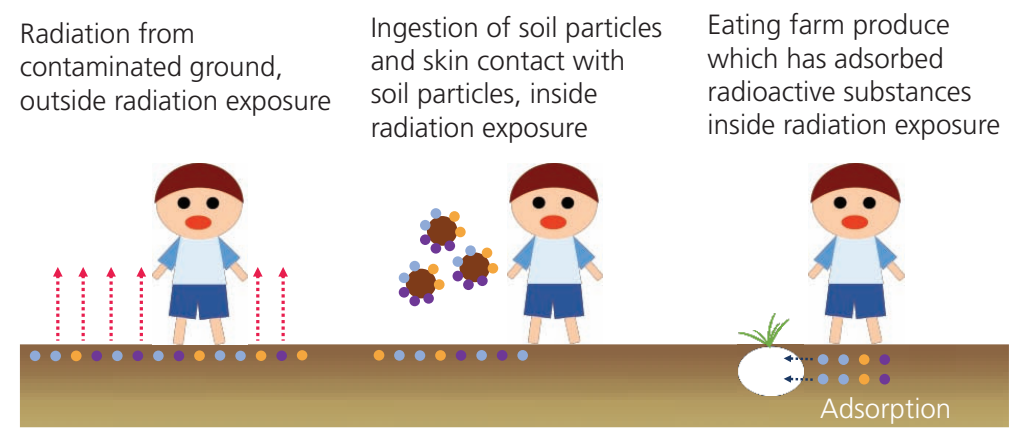

Figure 1. Pathways through which a person is exposed to radiation from contaminated ground

In experimental investigation, the handling of radioactive substances is highly dangerous. In addition, a long experimental period is required when radioactive substances with longer halflives are handled (Vardon and Heimovaara, 2015). Moreover, there have been no analytical investigations to estimate the transportation behaviour of radioactive substances in the soils. Therefore, this study includes a term for considering the half-life of radioactive substances in the advection-dispersion equation to estimate analytically the transportation behaviour of radioactive substances in the soil. The transportation behaviour of the radioactive substances in the specified soils obtained from this improved equation is compared with the density of the radioactive materials measured at the field site. Furthermore, the influence of various soil properties on the transportation behaviour of radioactive substances in the ground is investigated analytically by applying this improved equation.

\section{Provision for radioactive substances in the ground}

\section{Radiation exposure pathway from contaminated} ground by radioactive substances

There are three pathways by which a person can be exposed to radiation from contaminated ground. These are the pathway of radiation exposure caused by the contaminated soil, the pathway of radiation exposure caused by possible ingestion or skin contact with scattered soil particles and the pathway of internal radiation exposure caused by eating farm produce that has adsorbed radioactive substances from contaminated ground (Tsukada et al., 1998), as shown in Figure 1.

According to a study that compared radiation exposure resulting from contaminated soils with the internal radiation exposure caused by ingestion or skin contact with scattered soil particles, the latter represents $95 \%$ of all radiation exposure (Ministry of Education, Science and Culture, 2011b). The Ministry of Health, Labour and Welfare has been performing dosimetry of the internal radiation caused by consumption of farm produce that has absorbed radioactive substances from contaminated soil. Food monitoring was performed from March to August 2011.
According to the results, the dosage in the farm produce was $0 \cdot 041-0 \cdot 135 \mathrm{mSv}$ over 6 months (Ministry of Health, Labour and Welfare, 2011), whereas a person would receive a dosage of approximately $1.2 \mathrm{mSv}$ by consuming dairy products over 6 months (UNSCEAR, 1988). Therefore, the soil can be evaluated as safe in terms of human health.

\section{Classification of countermeasures for contaminated ground from radioactive substances}

Reduction methods for the pathways of radiation exposure resulting from contaminated soil can be classified into four measures (Tsukada et al., 1998), as shown in Figure 2. These are system management, original position management, original position processing and removal of the contaminants. Figure 2 shows a schematic diagram of the classification of the four measures. Furthermore, each measure is outlined and discussed in turn below.

System management is a method of reducing dosage by forbidding people to enter the contaminated area. Prohibiting entrance to a contaminated area limits the measures required by designating a remediated evacuation zone and caution area. The technical degree of difficulty is low; nevertheless, land use is extremely limited. Since the residents of a contaminated area must be moved, the mental and financial burdens of each refugee, as well as the social cost and limitations on land use, can become significant.

Original position management is a method of reducing the dosage that occurs as a result of falling air radiation contamination by covering the ground surface and reverse tillage. The technical degree of difficulty of this method is also low, and the cost of implementation is inexpensive. However, long-term management is necessary, and land use must be limited in a controlled manner.

Original position processing is a method of decreasing the concentration of radioactive substances at the field site, which does not involve removing the contaminated ground. Such methods include soil disturbance, removal by water using paddy fields and phytoremediation. 


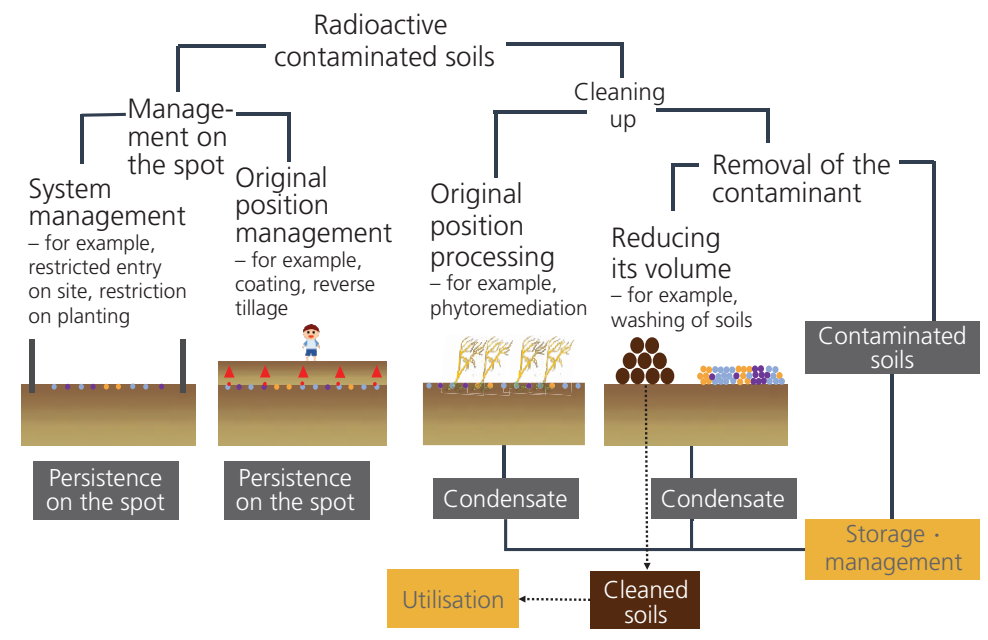

Figure 2. The classification of reduction methods for contaminated grounds

The removal of contaminants is a method of decreasing the concentration of radioactive substances at the field site by removing contaminated ground. This method consists of three phases: removing the contaminated ground, volume reduction and storage. After removing contaminated ground, there is a configuration for storing the contaminated ground without reducing the volume and a configuration for storing the concentrate that is composed of the radioactive substances removed from the contaminated ground.

These measures differ in terms of their direct costs, period required for implementation, technical completeness of the solution and indirect costs.

\section{The present situation regarding decontamination of contaminated ground}

The guidelines used for decontaminating ground contaminated by radioactive substances designate areas such as schoolyards, gardens, parks and farmlands as locations to consider for decontamination. Generally, decontamination is conducted until the local radiation dosage rate is less than $0.23 \mathrm{mSv} / \mathrm{h}$ (Ministry of the Environment, 2013).

The procedure of decontaminating begins with measuring the local radiation levels before the start of decontamination in order to be able to confirm the effects of the decontamination process. Next, decontamination is conducted at a hotspot where the ground has a higher level of contamination than the girth of the storm drainage containing the radioactive substances. This occurs after covering the decontamination locations with tarpaulin to prevent the spread of radioactive substances. Several typical examples of hotspots include hollows in the ground, water pockets, ditches and areas under trees. When decontamination is insufficient, methods such as covering the surface of the ground, reverse tillage and removing the surface of the ground are used to decrease the effects of radiation.
Covering the ground surface is an approach used whereby the upper soil layer containing the radioactive substances is covered with normal soil. Reverse tillage is a way to cover the ground, which is approximately $10 \mathrm{~cm}$ thick, by replacing it with a $20 \mathrm{~cm}$ thick layer of normal soil, thereby containing radioactive substances. A sufficient shielding effect when removing the ground is achieved by removing the ground up to an approximate depth of $5 \mathrm{~cm}$.

The advantages of the two previous methods ensure that the contamination from the atmosphere will not increase and the radioactive substances will not spread once covered with a soil layer. Additionally, contaminated soils will not appear, nor will there be radical contamination since the radioactive substances exist in the ground. Therefore, the ground surface layer can be removed manually, or heavy machinery can be used, as shown in Figure 3.

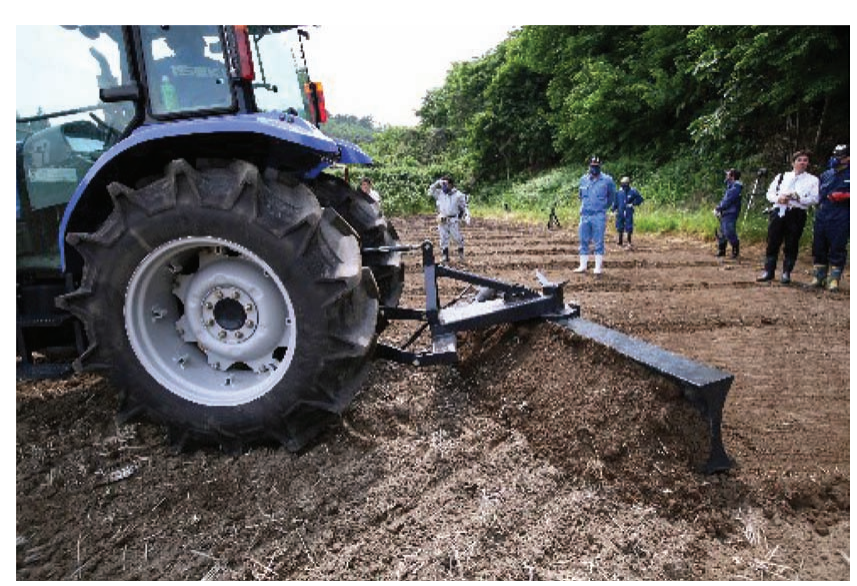

Figure 3. The decontamination of grounds 


\section{Behaviours of radioactive substances in the ground}

The radioactive substances that have fallen on the ground experience four situations: concentration decrement by radioactive decay, movement in the soil, scattering and flowing due to weather and absorption by plants (Kato et al., 2012).

\section{Concentration decrement by radioactive decay}

Concentration decrement by radioactive decay depends on the radioactive half-life. Figure 4 shows the time courses for the survival rates of iodine-131, caesium-131 and caesium-137. The radioactive half-life of iodine-131 is almost $8 \mathrm{~d}$. Therefore, its level of contamination becomes negligible relatively quickly. Caesium-131, whose radioactive half-life is approximately 2 years, disappears after approximately 15 years. However, the radioactive half-life of caesium-137 is almost 30 years, requiring long-term scrutiny.

\section{Movement in the soil}

The existing form of radioactive caesium and the soil properties are important factors to consider. Radioactive caesium in soil exists as a monovalent cation, $\mathrm{Cs}^{+}$. There are clay minerals and organic substances that have a significant amount of negative electric charge on their surface. Therefore, radioactive caesium is adsorbed on them in a similar manner to other heavy metals and positive ions (Nguyen et al., 2017).

\section{Adsorption of radioactive caesium using a cauterisation agent}

Most organic substances, such as animal and plant matter, that enter the soil are broken down into water and carbon dioxide gas through the action of soil, animals and microbes. A portion exists in the soils as a difficult, degradable high-molecular-weight compound called a cauterisation agent. This cauterisation agent has a carboxyl group, $-\mathrm{COOH}$, on its surface. It also has a negative charge based on dissociating a hydrogen ion, $\mathrm{H}^{+}$. Consequently, the cauterisation agent becomes the adsorbent of radioactive caesium. However, radioactive caesium, which is

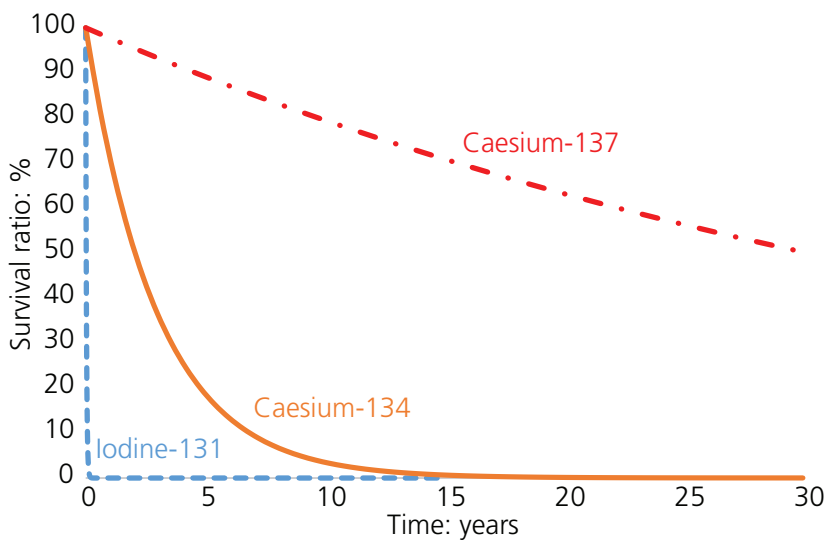

Figure 4. Circumstances of decay by radioactive half-life absorbed with a cauterisation agent, is permuted easily by other cations because this absorption is a weak combination through the hydrated water surrounding the caesium ion. The cauterisation agent is an adsorbent that maintains the high mobility of radioactive caesium. Radioactive caesium adsorbed with the cauterisation agent gradually transitions to a selective high adsorbent through a repetitive adsorption and desorption process.

Adsorption of radioactive caesium with frayed edge site A 2:1 type layer silicate is one of the mineral constituents of clay particles. The silicate holds negative charges on a silicon tetrahedral sheet. Mica holds a larger number of negative charges than potassium ion $\left(\mathrm{K}^{+}\right)$and occupies a six-membered ring. Consequently, other negative charges can enter the layer as the layer interval already has been strongly closed.

A distal portion of the layer swells in the state that maintained the seat structure by aeration. As a result, potassium ion is released from a layer interval of a circumferential section and a hydrated positive ion is held in a layer interval. The wedge-shaped border section of the swelling and non-swelling layers is called the frayed edge site. In a frayed edge site, a hydrated positive ion is released by spatial limitation. Then, caesium ions, whose hydration energy is the lowest and whose shape is fit for a sixmembered ring, is adsorbed strongly to the frayed edge site, as can be seen in Figure 5.

\section{Scattering and flowing out by weathering}

As mentioned earlier, radioactive caesium is adsorbed by clay mineral and organic substances and then tends to take root on the field site. In addition, it is found that radioactive substances that are adsorbed do not dissolve in water easily (Ministry of the Environment, 2011c). For this reason, there are no incidents where radioactive caesium has been detected in surface water and/or groundwater. However, there is research that indicates that some movement of radioactive caesium occurs. The radioactive substances that are adsorbed by the soil could be estimated to be re-transferred near the ground surface, transitioned to surface water and groundwater and re-entrained into the air (Kato et al., 2012).

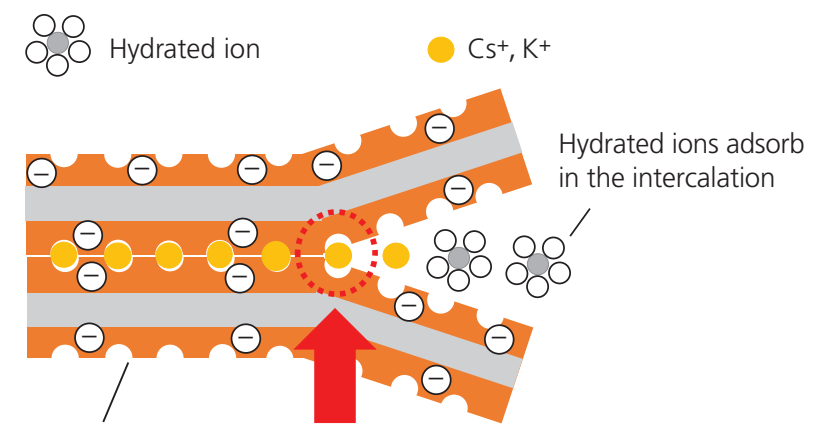

Negative charges

on the silicon

Frayed edge site

tetrahedral sheet 


\section{Removal near the ground surface}

The weathering effect occurs when material flows out of soils near the ground surface. It is a primary factor for the removal or material near the surface of the ground. The results relating to radioactive concentrations in rice fields and farms in Japan after the nuclear test were monitored and analysed by Komamura et al. (2006). It was found that the radioactive half-life of caesium-137, which is considered to be subject to the weathering effect, was $15 \cdot 9$ years in a rice field and $18 \cdot 4$ years in a farm.

\section{Transition into surface water and groundwater}

The radioactive substances that are adsorbed by soils flow into rivers due to heavy rain and snowmelt, and then they are drained downstream. The radioactive substances that are strongly adsorbed by clay do not dissolve easily in water. Accordingly, radioactive substances were detected in surface water and groundwater in many cases. Examinations were performed in aquatic environments, such as rivers and lakes, based on the comprehensive monitoring plan from September 2011 established by the Ministry of the Environment (2011c). Radioactive substances were not detected in the surface water at Fukushima.

At the two field sites within $20 \mathrm{~km}$ of the Fukushima Daiichi Nuclear Power Plant, radioactive substances measured at levels of 1-2 Bq/1 were detected in the groundwater. However, no other sites had measurable activity (Ministry of the Environment, 2011c). On the other hand, the outflow and spread of the radioactive substances by soil draining of the downstream sector were estimated to result in a volume of outflow from forests of approximately $0.3 \%$ of the volume of soil inflow to the forest a year after occurrence (National Institute of Advanced Industrial and Technology, 2012).

\section{Re-entrainment into air}

Soils that have adsorbed radioactive substances move as dust particles. Otherwise, it is assumed that radioactive substances that have fallen onto plants have moved. From the results of the dust monitoring in Fukushima by the Ministry of Education, Culture, Sports, Science and Technology, radioactive substances were detected after January 2012 (Ministry of the Environment, 2013). Moreover, a trial calculation of the radiation dosage that the human body received by inhaling cedar pollen was carried out. According to this trial, the dosage rate was $0 \cdot 000192 \mu \mathrm{S} / \mathrm{h}$ (Forestry Agency, 2011), which is extremely small, presenting a near-zero risk to humans.

\section{Absorption by plants}

There are two possibilities for radioactive substances released into the atmosphere to be transferred to plants. One pathway is directly from the atmosphere, which is called the directly absorbed course (Mohammad, 2015). The other pathway is when radioactive substances that have fallen on the ground are absorbed into the root of the plant, which is called the root absorption course, as shown in Figure 6.

Following the Fukushima Daiichi Nuclear Power Plant accident, the directly absorbed course was a significant influence when the

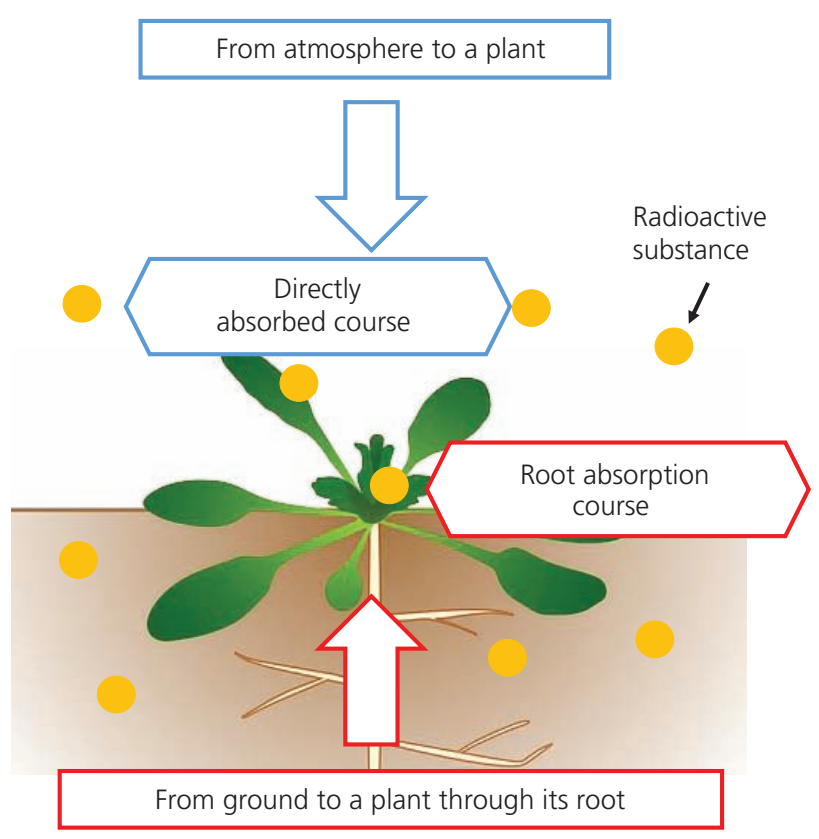

Figure 6. The courses of the transfer of radioactive substances into a plant

quantity of radioactive substances was large. Afterwards, the root absorption course became more influential. Furthermore, since pollution of the plants by the directly composed course was dependent on the quantity of radioactive substances, the period was relatively short. In contrast, most radioactive substances remained in the soil for a long period of time because of their strong absorption characteristics. Therefore, radioactive pollution of the plants by the root absorption course is expected to last several decades owing to the long radioactive half-life.

The quantity of absorption by the root absorption course is dependent on the concentration of the radioactive substances and the transition coefficient. The transition coefficient is a parameter measuring the tendency of radioactive substances to transfer from soil to plants and can be written as

concentration of the radioactive

transition $=$ substance in edible part of plant coefficient $=\overline{\text { concentration of the radioactive }}$

1. substance in the soil

The transition coefficient differs according to the type of crop. The average values for polished rice and potato are 0.0005 and 0.011 respectively (Ochiai et al., 2009).

\section{Seepage and advection-dispersion analysis for radioactive substances}

Scattering and flowing out by weather, as well as absorption by plants, have extremely small values. Moreover, in order to evaluate pure transportation behaviour in the soil, only the concentration 
decrement due to radioactive decay and movement in the soil are a focus of this study. Generally, moving substances in the soil analysis are modelled by the advection-dispersion equation (Bharat, 2014). The results for movement of radioactive substances that can be derived by this equation were added to a term that considers radioactive half-life. The velocity distribution and the volumetric water content distribution, as calculated by the seepage analysis, were necessary to conduct the advection-dispersion analysis. Therefore, a seepage and advection-dispersion analysis that considers the radioactive half-life was carried out in this study.

\section{The seepage equation}

In the steady state, the general management for two-dimensional seepage (Desai et al., 2011) can be expressed as the equation

2.

$$
k_{x} \frac{\partial^{2} H}{\partial x^{2}}+k_{y} \frac{\partial^{2} H}{\partial y^{2}}+Q=0
$$

where $H$ is the total head, $k_{x}$ is the hydraulic conductivity in the $x$ direction, $k_{y}$ is the hydraulic conductivity in the $y$ direction and $Q$ is the applied boundary flux. Further, the hydraulic conductivity is estimated to be equal in the $x$ and $y$ directions.

The advection-dispersion equation, which includes the radioactive half-life, can be written as

$$
\theta \frac{\partial C}{\partial t}+\rho_{\mathrm{d}} \frac{\partial S}{\partial C} \frac{\partial C}{\partial t}=\frac{\partial C}{\partial x_{i}}\left(\theta \boldsymbol{D}_{i j} \frac{\partial C}{\partial x_{j}}\right)-U_{i} \frac{\partial C}{\partial x_{i}}
$$$$
3 .
$$$$
-\lambda\left(\theta C-S \rho_{\mathrm{d}}\right)
$$

where $\theta$ is the volumetric water content, $C$ is the concentration of the radioactive substance, $\rho_{\mathrm{d}}$ is the dry density of the soil, $\boldsymbol{D}_{i j}$ is the dispersion tensor, $U_{i}$ is the Darcian velocity, $t$ is the time, $\lambda$ is the decay coefficient and $S$ is the adsorption.

\section{The dispersion tensor $D_{i j}$}

The dispersion tensor $\boldsymbol{D}_{i j}$ is shown as the equation

4. $\boldsymbol{D}_{i j}=\alpha_{\mathrm{T}}\|V\| \delta_{i j}+\left(\alpha_{\mathrm{L}}-\alpha_{\mathrm{T}}\right) \frac{v_{i} v_{j}}{\|V\|}+D^{*} \tau \delta_{i j}$

where $\alpha_{\mathrm{L}}$ is the longitudinal dispersivity, $\alpha_{\mathrm{T}}$ is the transverse dispersivity, $v_{i}$ is seepage velocity, $\|V\|$ is the norm of actual flow, $D^{*}$ is the coefficient of molecular diffusion of the radioactive substance, $\tau$ is the tortuosity and $\delta_{i j}$ is Kronecker delta (Mohammad, 2013).

Dispersion is a phenomenon where substances spread in the flow direction. Dispersivity is divided into longitudinal and transverse dispersivities. Longitudinal dispersivity is the degree of concentration that is a blurred antecedent to the water velocity in the direction of the water flow. In contrast, transverse dispersivity is the degree of concentration that is blurred towards the direction at right angles to the water flow (Mohammad, 2012a, 2012b), as can be seen in Figure 7. When the analysis scale is approximately $1 \mathrm{~m}$, the vertical dispersivity is determined to be $0 \cdot 01$. In addition, when the analysis scale is approximately $10 \mathrm{~m}$, the longitudinal dispersivity is at $0 \cdot 1$ (National Institute for Environmental Studies, 2012). Moreover, transverse dispersivity is one-tenth of longitudinal dispersivity (Gelhar and Axness, 1983). Advection is a phenomenon of concentration that is mutated by this motion. The molecules move consistently unless they are at the molecular absolute zero point.

\section{The decay coefficient $\lambda$}

The decay coefficient is a parameter for expressing the phenomenon of the concentration decrement as a result of the radioactive half-life. This phenomenon is expressed by an exponential function and can be written as

5. $C=C_{0} \mathrm{e}^{-\lambda t}$

where $C_{0}$ is the initial concentration.

The ratio of the concentration to the initial concentration is then $1 / 2$ for the case where the time is the radioactive half-life, $T_{1 / 2}$. This can be seen in the equation

6. $\frac{C}{C_{0}}=\frac{1}{2}=\mathrm{e}^{-\lambda T_{1 / 2}}$

This leads to the equation

7. $\lambda=\frac{\ln (2 \cdot 0)}{T_{1 / 2}}$

\section{The adsorption $S$}

The adsorption is calculated by using the linear adsorption isotherm with the distribution coefficient $K_{\mathrm{d}}$, as shown in the equation

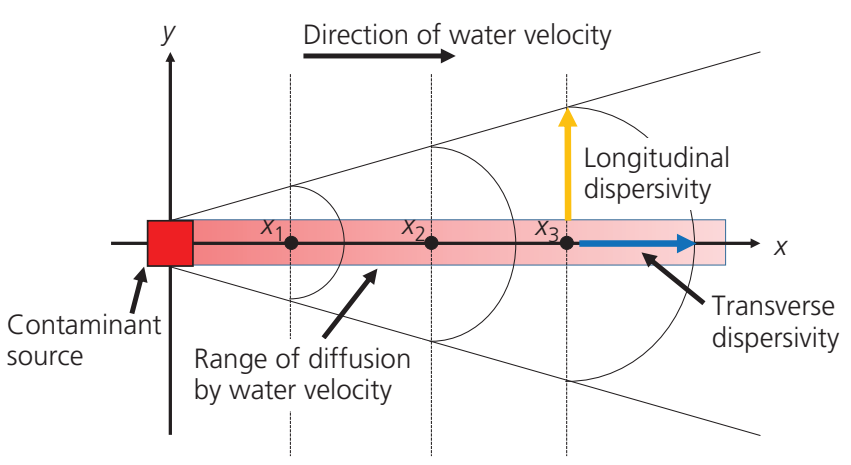

Figure 7. Concept of dispersivity. $x_{n}$, distance of contaminant diffusion by water velocity when time $t$ passes 
8. $S=K_{\mathrm{d}} C$

The distribution coefficient is an index for expressing the hydrophobicity and the transitivity of a chemical substance. This index is determined by the radioactive substance and the soil. Moreover, it is the slope of the linear adsorption isotherm.

\section{Validity of the seepage and advection-dispersion analysis}

The analysis results were compared with the concentration distribution measurement results for caesium-137 from the area affected by the accident at the Fukushima Daiichi Nuclear Power Plant in order to validate the appropriateness of this analysis method.

\section{The measurement results}

The measurement results were recorded by Saito et al. (2012) in the Suginoura meeting place, which is approximately $70 \mathrm{~km}$ from the Fukushima Daiichi Nuclear Power Plant. The measurements were taken three times, after 9, 18 and 20 months. A scraper plate, the use of which is accepted as the standard sampling method of the depth direction by the International Atomic Energy Agency, as shown in Figure 8, was used for soil sampling. The use of a scraper plate is a method for taking samples by stripping soil off in small amounts from the ground surface and collecting it. Soil samples were taken from the ground surface at depths of $0 \cdot 5,0 \cdot 5-1 \cdot 0,1 \cdot 0-1 \cdot 5,1 \cdot 5-2 \cdot 0,2 \cdot 0-3 \cdot 0,3 \cdot 0-4 \cdot 0$ and $5 \cdot 0-8 \cdot 0 \mathrm{~cm}$. Gamma beam spectrum analysis with a germanium semiconductor detector was conducted for measuring caesium-137.

Additionally, the soil at this field site was studied by particle size measurement using sieving and laser-diffraction-type equipment to measure the particle size distribution. Sieving was carried out for particle sizes greater than $425 \mu \mathrm{m}$. The laser-diffraction-

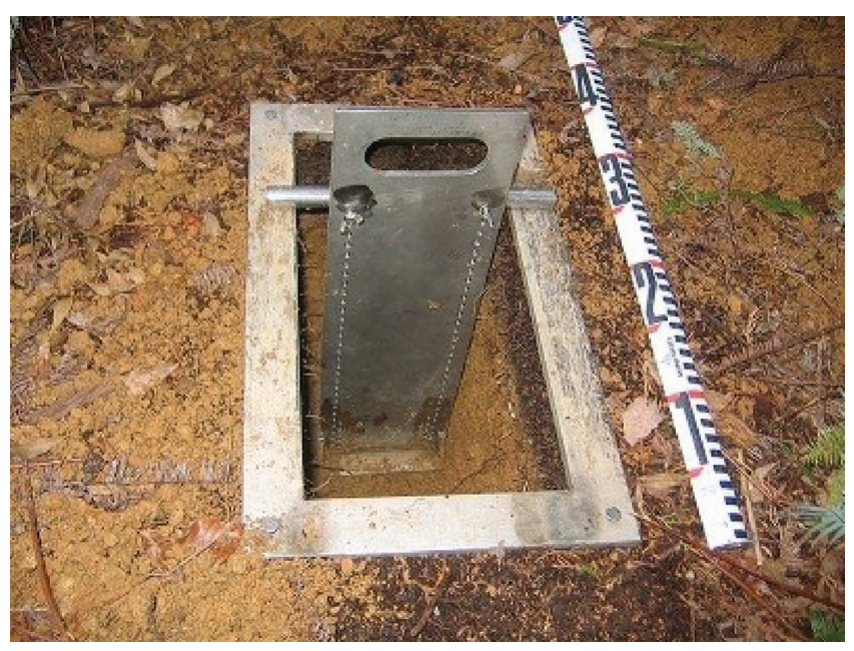

Figure 8. The method of obtaining soil samples by using a scraper plate type equipment was used for sizes less than $425 \mu \mathrm{m}$. Figure 9 shows the measurement results. According to these results, the concentration at 9 months was distributed exponentially from approximately $10000 \mathrm{~Bq} / \mathrm{kg}$ near the ground surface. The concentration near the ground surface reduced by approximately $1000 \mathrm{~Bq} / \mathrm{kg}$ over 9 months because the concentration at 18 months was almost $9000 \mathrm{~Bq} / \mathrm{kg}$. This can be attributed to the four actions described earlier regarding the movement of radioactive substances in the soil. However, caesium-137 remained at a depth of approximately $5 \mathrm{~cm}$ for all periods.

Since the decreasing tendency is similar even if time passes, this point might occasionally be disordered by strong rain or factitious external force. Relative to other points, the concentration was distributed in an approximately flat profile until reaching a significant depth. However, for the entire set of measurement results at the field site, the concentration in the depth direction was distributed similarly to an exponential function. The particle size measurement revealed that this point had a particle size of $5-75 \mu \mathrm{m}$ in the silt layer.

\section{Conditions for analysis}

One-dimensional seepage and advection-dispersion analysis, which considered the radioactive half-life, was carried out in order to evaluate quantitatively the concentration distribution in the depth direction in the soil. The section chosen for analysis was the silt layer. Table 1 shows the typical values for silt. The hydraulic conductivity $k$ is obtained from the Japanese Geotechnical Society (2002). The distribution coefficient of silt is based on the work of Inoue and Morisawa (1976). However, the coefficient of molecular diffusion and the initial concentration of caesium-137 at this point were proven by Bossew and Kirchner (2004). The radioactive half-life used for caesium-137 was $30 \cdot 2$ years and the longitudinal dispersivity was assumed to be $0.01 \mathrm{~m}$. These values for caesium-137 are shown in Table 2.

\section{Appropriateness of the seepage and advection-dispersion analysis considering radioactive half-life in measurement results}

Figure 9 shows the measurement and analysis results of the concentration distribution of caesium-137 in the depth direction. The analysed values approach the measured values at each depth. Likewise, the tendency of the decreased concentration in the analysis result is close to the measured result. Furthermore, the values are the same over the passage of time. Therefore, seepage and advection-dispersion analysis, which considered the radioactive half-life, can reproduce the concentration distribution at the field site.

\section{Influence of soil properties on transportation in the soils}

The seepage and advection-dispersion analysis, which considered the radioactive half-life, was carried out with various soil properties. The results are shown to contour the figure of concentration and the concentration distribution in the depth 

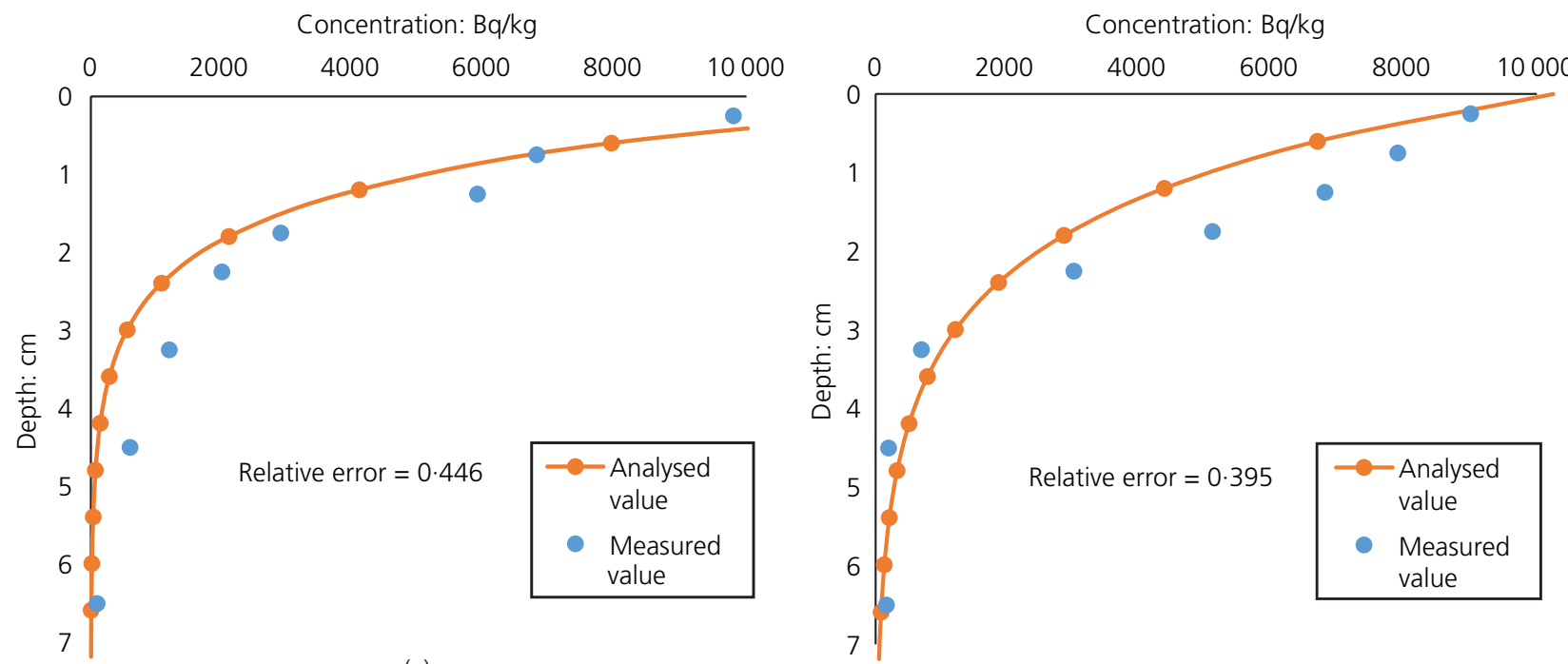

(a)

(b)

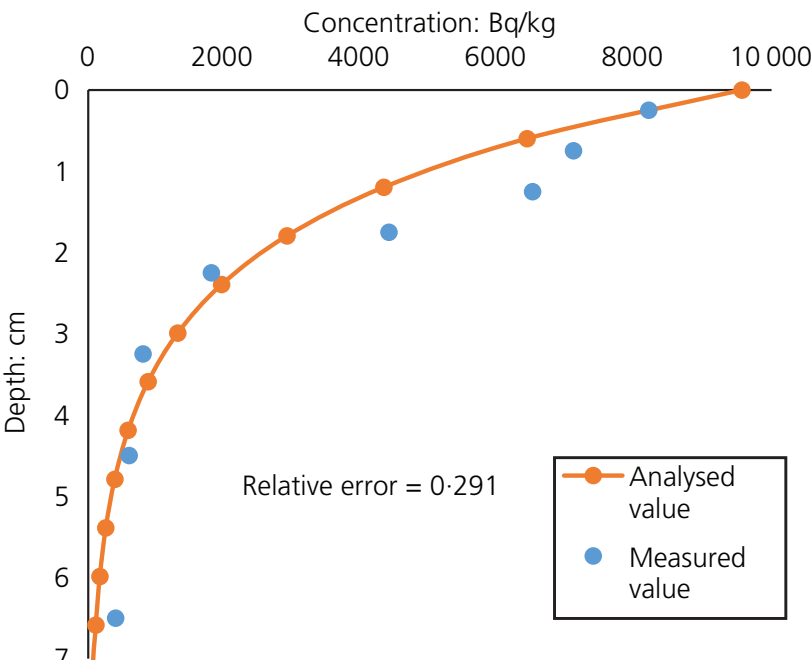

(c)

Figure 9. Analysis results and the measurement results of caesium-137 concentration distribution in depth direction: (a) 9 months' progress; (b) 18 months' progress; (c) 20 months' progress

direction at a certain point. The differences in the behaviour of the radioactive substance in the soil were evaluated.

\section{Analysis conditions}

The section for analysis is shown in Figure 10. The transportation behaviours were evaluated at a local section where a difference in water head occurred. Furthermore, the section was saturated so that it was possible to evaluate the most critical situation where caesium-137 had fallen onto the ground surface. The boundaries

Table 1. Parameters for silt

$\begin{array}{lc}\text { Hydraulic conductivity, } k: \mathrm{m} / \mathrm{s} & 1.0 \times 10^{-6} \\ \text { Volumetric water content, } \theta & 0.4 \\ \text { Dry mass density of soil, } \rho_{\mathrm{d}}: \mathrm{g} / \mathrm{m}^{3} & 1.2 \times 10^{6} \\ \text { Distribution coefficient, } K_{\mathrm{d}}: \mathrm{g} / \mathrm{m}^{3} & 8.0 \times 10^{-3}\end{array}$

were coloured blue on a section to represent a drainage condition. The soils for analysis are selected as silt, sand and clay. There are four cases: case 1 is the silt layer; case 2 is the sand layer; case 3 is the clay layer for all ranges of the section used for analysis; and case 4 is alternate layers of silt in section 1 and clay in section 2 .

Typical values are used for each parameter, as shown in Table 3. The hydraulic conductivities $k$ of sand and clay are in accordance

Table 2. Parameters for caesium-137

$\begin{array}{lc}\text { Radioactive half-life, } T_{1 / 2}: \text { years } & 30.2 \\ \text { Coefficient of molecular diffusion, } D^{*}: \mathrm{m}^{2} / \mathrm{d} & 7.2 \times 10^{-6} \\ \text { Initial concentration, } \mathrm{C}_{0}: \mathrm{Bq} / \mathrm{m}^{2} & 3.0 \times 10^{8} \\ \text { Dispersivity, } \alpha: \mathrm{m} & 0.01\end{array}$


Transportation behaviour of radioactive

substances in soils

Inazumi, Hashida and Ueyama

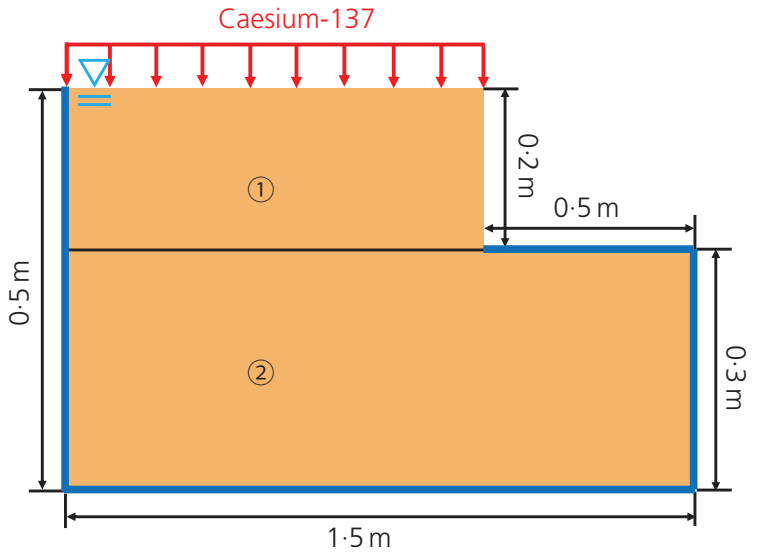

Figure 10. The section for analysis

Table 3. Parameters for silt, sand and clay

\begin{tabular}{|lccc|} 
& Silt & Sand & Clay \\
\hline Hydraulic conductivity, $k: \mathrm{m} / \mathrm{s}$ & $1.0 \times 10^{-6}$ & $1.0 \times 10^{-5}$ & $1.0 \times 10^{-7}$ \\
Volumetric water content, $\theta$ & 0.4 & 0.4 & 0.4 \\
Dry mass density of soil, $\rho_{\mathrm{d}}: \mathrm{g} / \mathrm{m}^{3}$ & $1.2 \times 10^{6}$ & $1.5 \times 10^{6}$ & $9.5 \times 10^{5}$ \\
Distribution coefficient, $K_{\mathrm{d}}: \mathrm{g} / \mathrm{m}^{3}$ & $8.0 \times 10^{-3}$ & $4.0 \times 10^{-3}$ & $1.0 \times 10^{-2}$
\end{tabular}

with the literature (Japanese Geotechnical Society, 2002). The Radioactive Waste Management Center (1990) studied the distribution coefficients of the sand and clay. Generally, the hydraulic conductivity $k$ of sand is larger than that of clay. The distribution coefficient $K_{\mathrm{d}}$ of sand is smaller than that of clay (Mohammad, 2012a, 2012b). However, caesium-137 was released in large quantities as a result of the accident at Fukushima. Typical values for caesium-137 above the initial concentration and the coefficient of the molecular diffusion at the field site were adopted. Therefore, Table 2 shows the additional transverse dispersivity. Since the height of the section used for analysis was $0.5 \mathrm{~m}$, the longitudinal dispersivity was determined as $0.1 \mathrm{~m}$. The transverse dispersivity was determined as $0.01 \mathrm{~m}$, one-tenth of the longitudinal dispersivity, as shown in Table 4.

\section{Transportation behaviours in each soil}

The streamline vector in the silt layer is shown in Figure 11. The contour lines expressing total head increased from blue to red. According to Figure 11, water movement occurred from areas of large total head to small - namely, from section 1 to section 2 . It was particularly strong from the right-hand edge of section 1 to the left-hand edge of section 2, which is confirmed for the other cases. The contours of the concentrations at 1,2, 5 and 10 years for all cases are shown in Figures 12, 13, 14 and 15, respectively. Caesium-137 is shown to exhibit advection and dispersion along the water flow for all cases.

In the silt layer, there was significant movement around the top of the slope. Therefore, caesium-137 reached almost $0 \cdot 1 \mathrm{~m}$ depth after
Table 4. Parameters for caesium-137

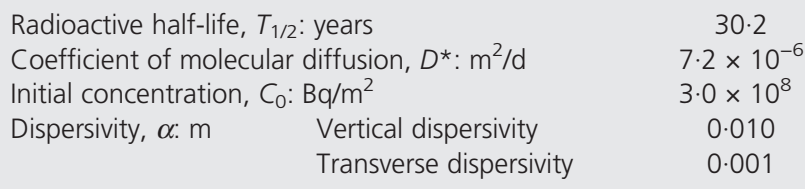

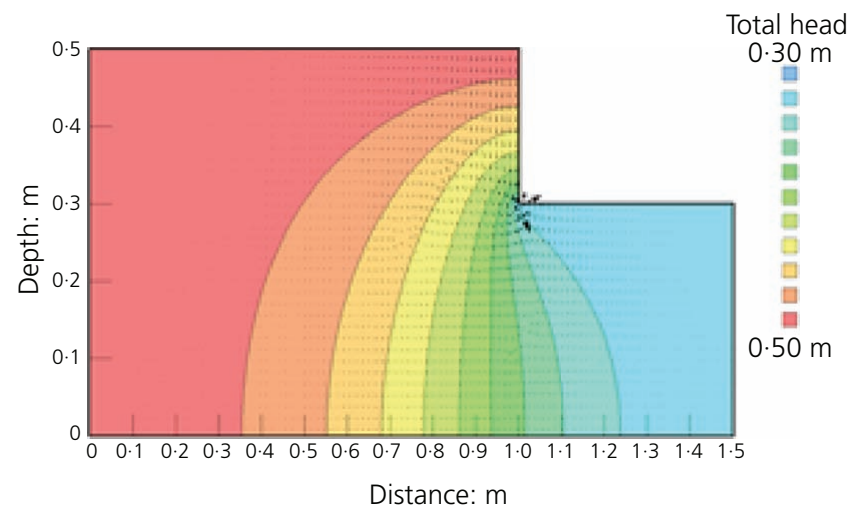

Figure 11. Flow vector in a silt layer

10 years. However, transportation in the sand layer was larger than that in the silt layer. Caesium-137 reached the ground surface of section 1. Moreover, because caesium-137 leached, the total concentration in the sand layer likely reduced. The result for reaching $0.1 \mathrm{~m}$ depth at 1 year is different from the measurement result at the field site. However, the transportation in the clay layer is the smallest of the three. There is a slight movement at the right-hand edge of the ground surface in section 1 . The movement was not caused by the hole. Accordingly, the concentration was relatively high at the ground surface.

Transportation of the alternate layer of silt and clay was different from the other cases. Migration was similar to case 2 until 2 years of progress had been recorded in the sand layer of section 1 . In practice, caesium-137 does not infiltrate within $9.5 \mathrm{~m}$ from the left edge over 10 years. Then, the concentration distribution in the depth direction of the $9.5 \mathrm{~m}$ field site was an alternate layer after 10 years of progress, compared with the sand layer, as shown in Figure 16. As Figure 16 indicates, the concentration of the alternate layer was distributed to a depth of $0.2 \mathrm{~m}$. However, the concentration in the sand layer reached a depth of over $0.2 \mathrm{~m}$. It follows that caesium-137 remained in the silt layer since transportation in the silt layer was unlikely to be noticeable, as mentioned earlier.

The levels of caesium-137 attained in each period are shown as the concentration distribution in the depth direction of the $0.7 \mathrm{~m}$ point, which is the centre of the section used for analysis, as shown in Figure 17. For all sections, the concentration at the ground surface reduced over time. For the silt layer, it was proven that caesium-137 could infiltrate while the concentration was decreasing. However, 
Environmental Geotechnics

Volume 6 Issue EG8
Transportation behaviour of radioactive

substances in soils

Inazumi, Hashida and Ueyama

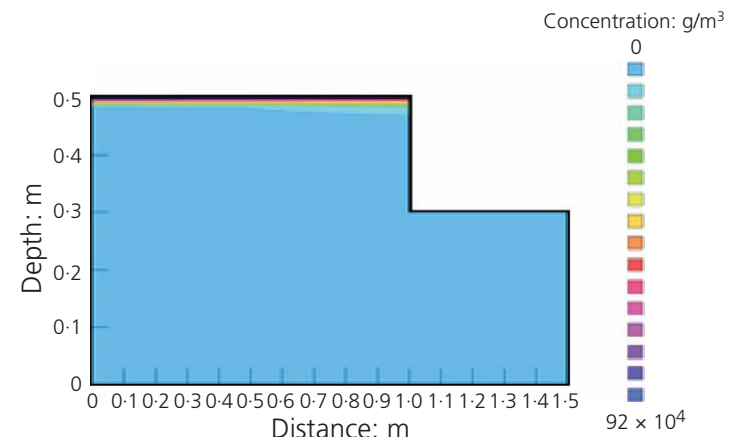

(a)

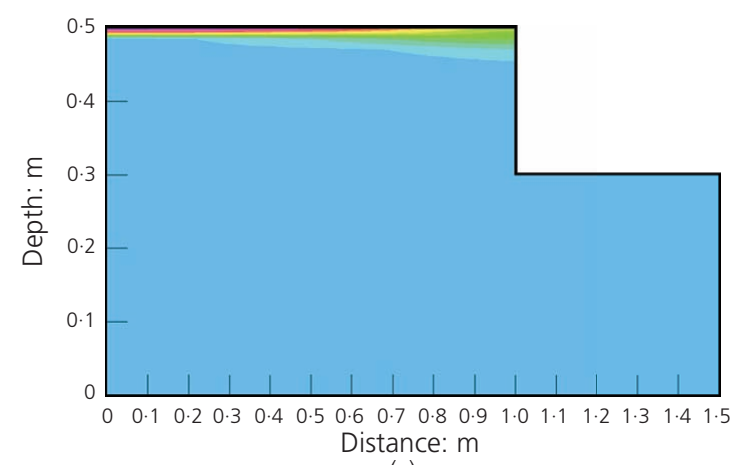

(c)

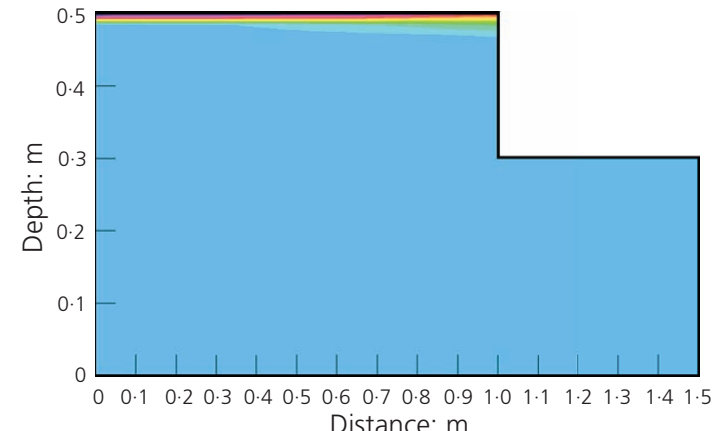

(b)

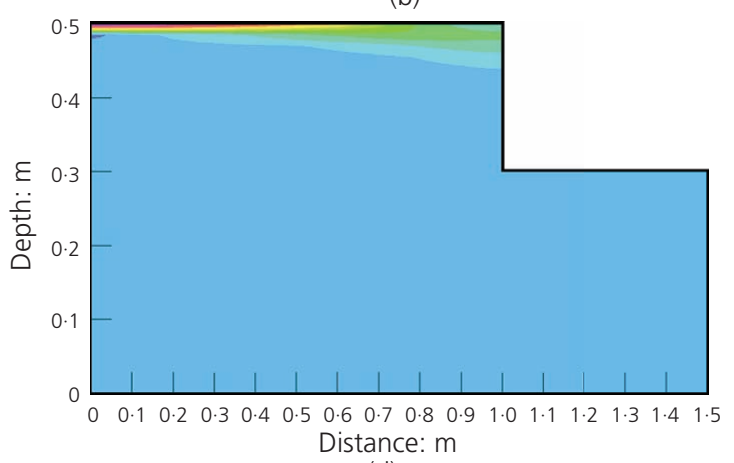

(d)

Figure 12. The transition of caesium-137 in a silt layer after (a) 1 year, (b) 2 years, (c) 5 years and (d) 10 years
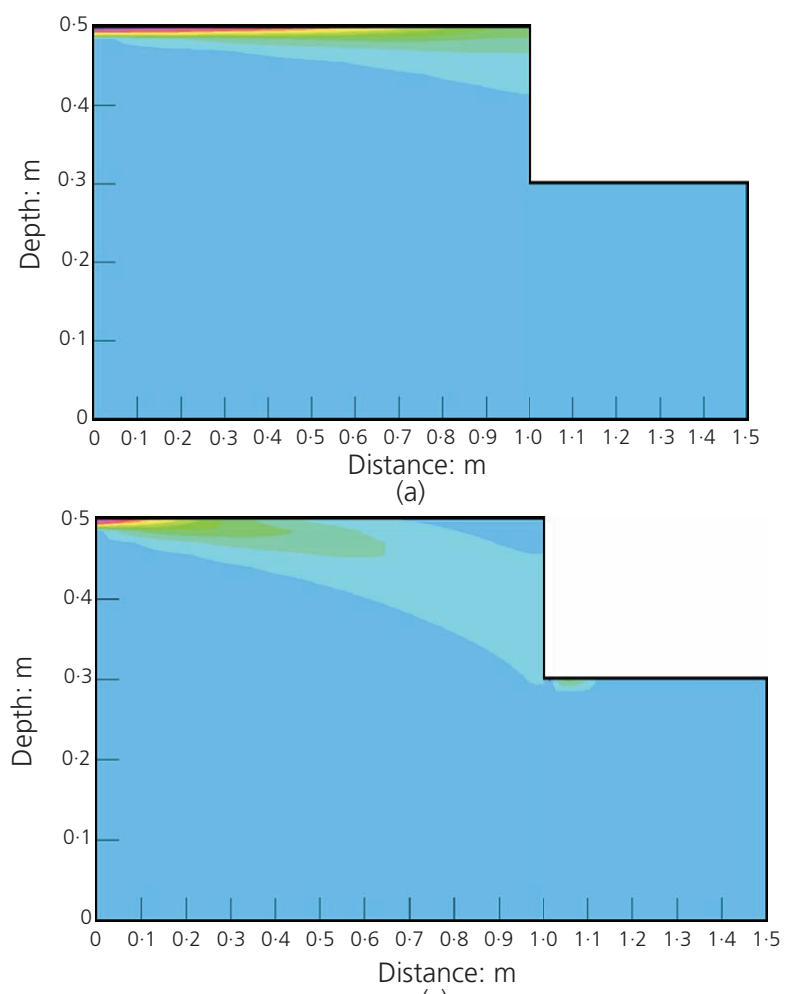

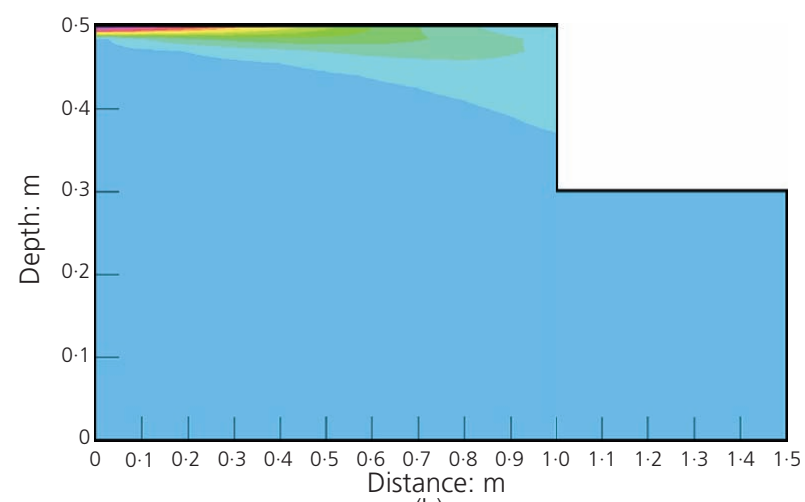

(b)

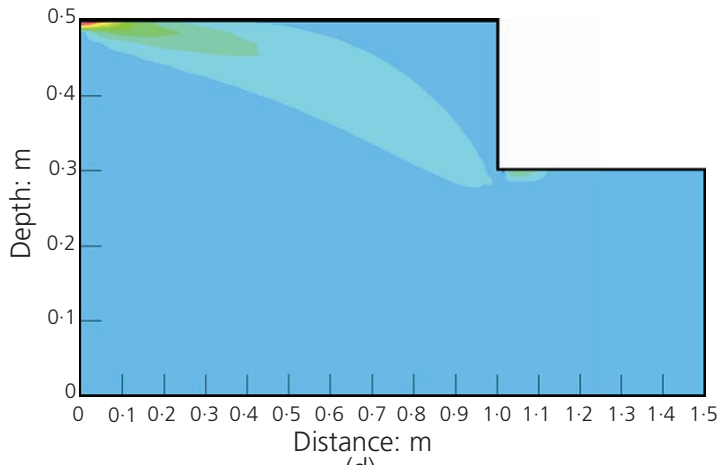

(d)

Figure 13. The transition of caesium-137 in a sand layer after (a) 1 year, (b) 2 years, (c) 5 years and (d) 10 years 


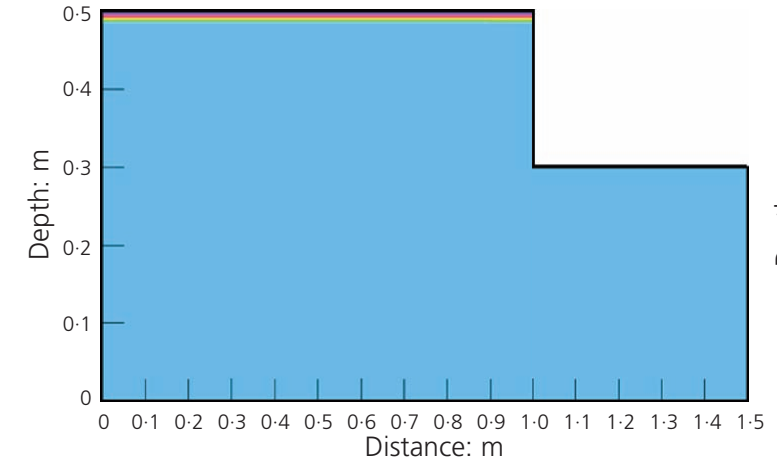

(a)

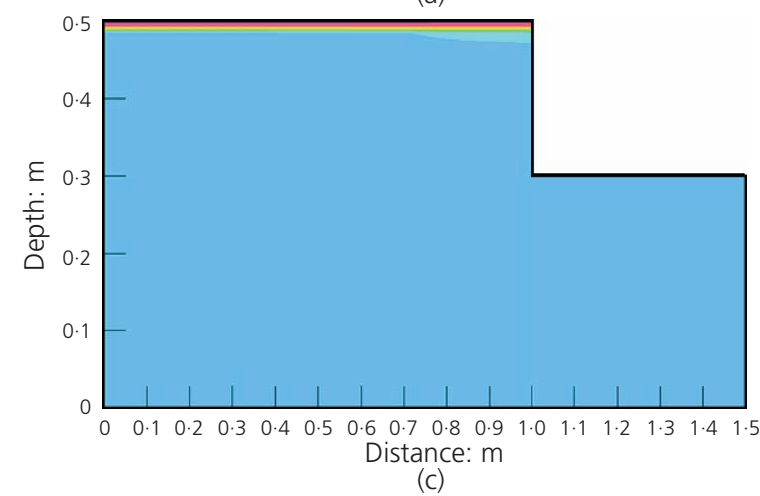

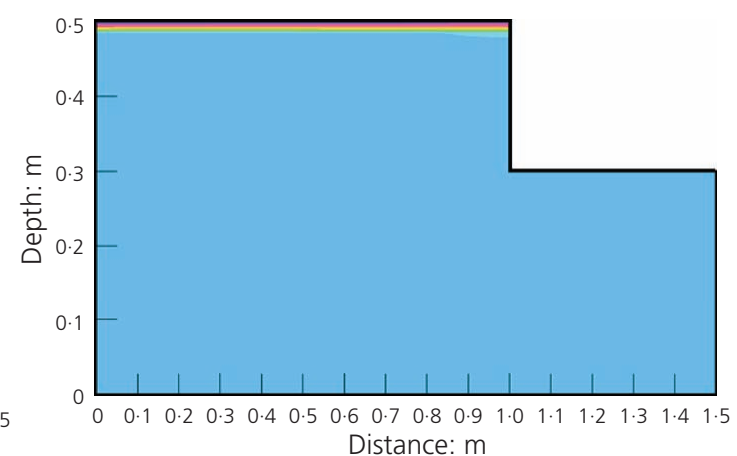

(b)

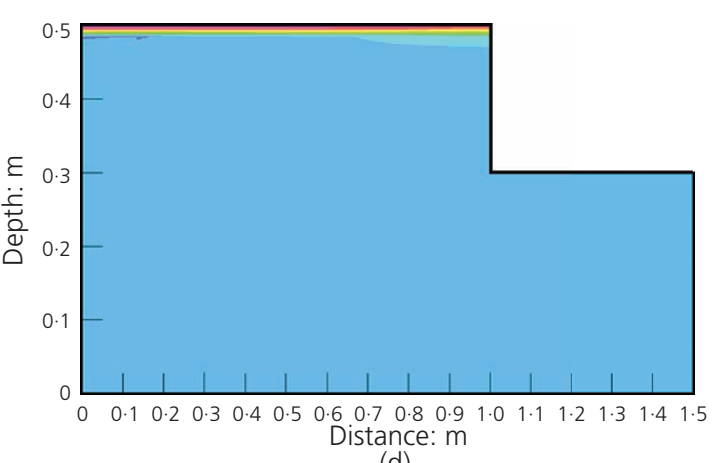

(d)

Figure 14. The transition of caesium-137 in a clay layer after (a) 1 year, (b) 2 years, (c) 5 years and (d) 10 years

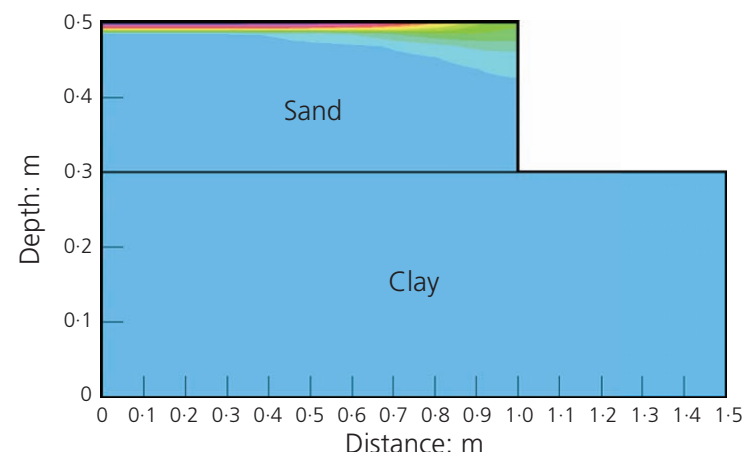

(a)

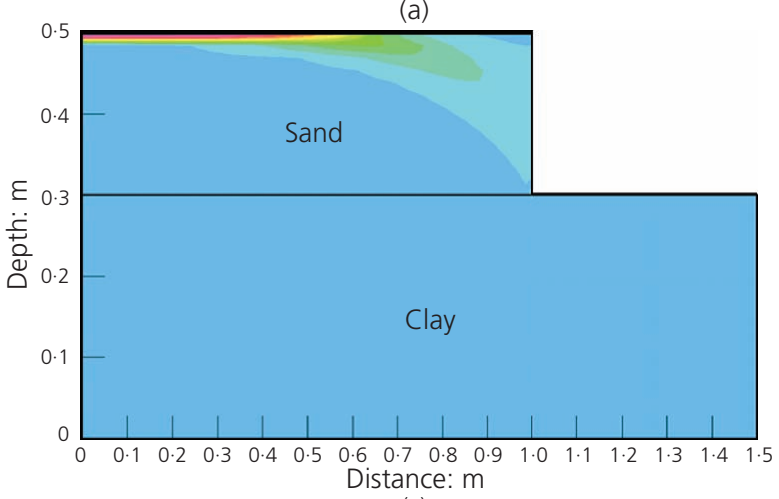

(c)

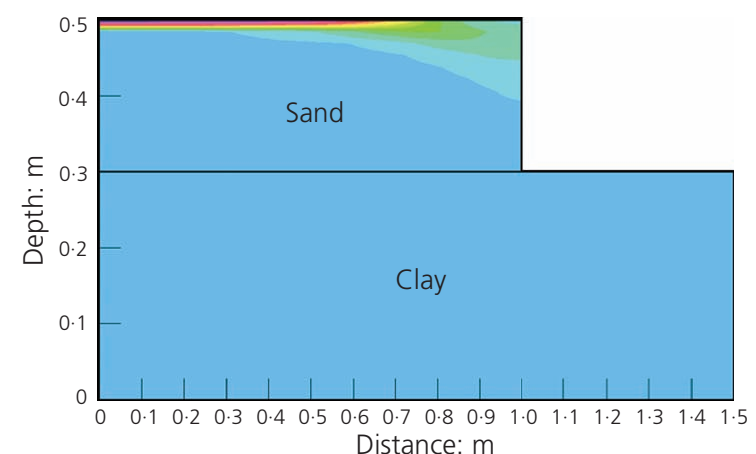

(b)

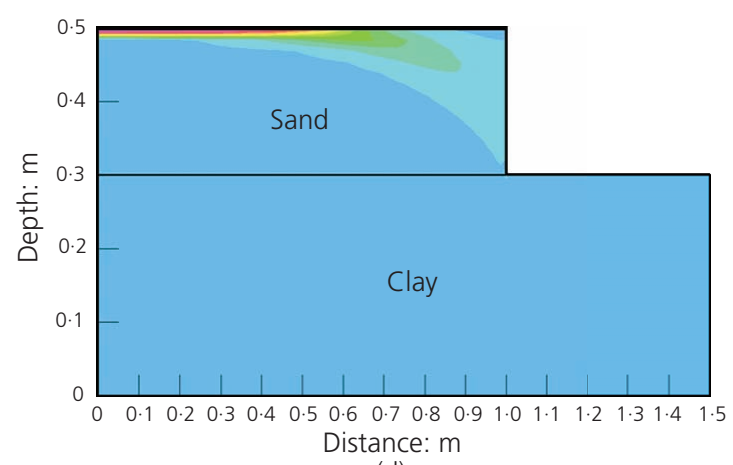

(d)

Figure 15. The transition of caesium-137 in an alternate layer after (a) 1 year, (b) 2 years, (c) 5 years and (d) 10 years 
Transportation behaviour of radioactive

substances in soils

Inazumi, Hashida and Ueyama

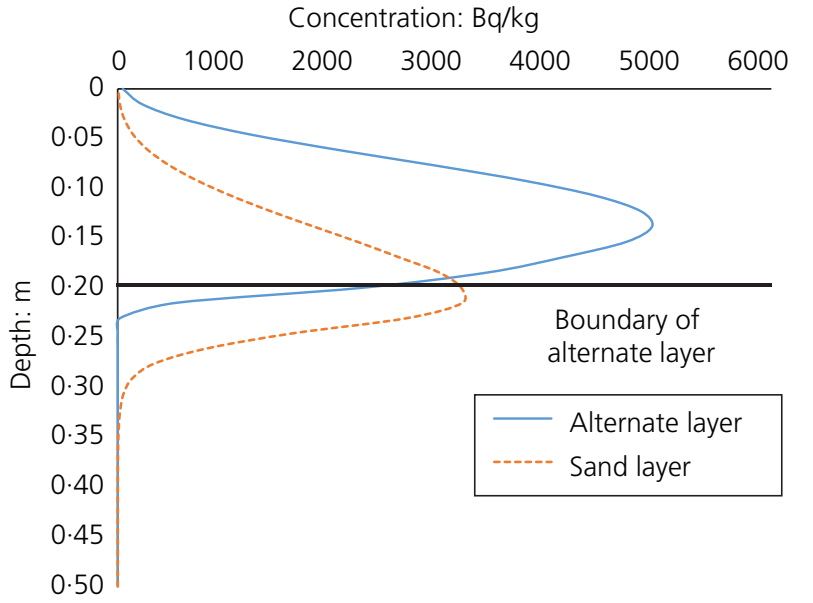

Figure 16. Concentration distribution at $9.5 \mathrm{~m}$ point in an alternate layer and a sand layer caesium-137 remained until it reached a depth of $0.05 \mathrm{~m}$ from the surface of the ground. In the sand layer, caesium-137 remained at ground level for 1 year. Based on 2 years of progress, the concentration has a shaped waveform. In other words, the most concentrated point appears inside the ground. In contrast, the ground surface was the most concentrated point even after 10 years. The concentration distribution in the alternate layer is a waveform with a time progression similar to that of the sand layer. Although transportation in the alternate layer was smaller than in the sand layer, caesium-137 reached a depth of $0.1 \mathrm{~m}$ after 10 years.

Difference between behaviours in sand and clay layers There is a significant difference between the behaviours of radioactive substances in sand and clay layers. The primary factor is an advection term that excels as an adsorption term. In brief, the hydraulic conductivity $k$ has a larger effect on transportation behaviours than the distribution coefficient $K_{\mathrm{d}}$. When the values of the hydraulic conductivity $k$ and the distribution coefficient $K_{\mathrm{d}}$

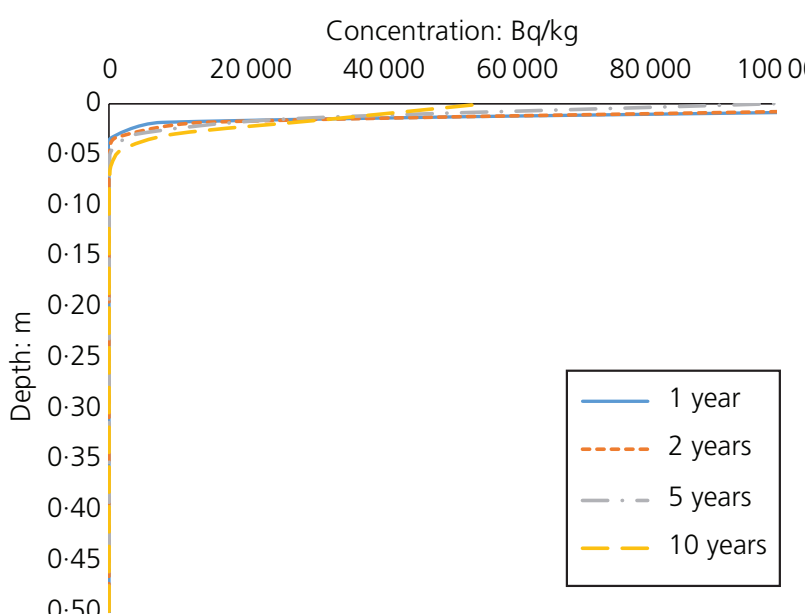

(a)

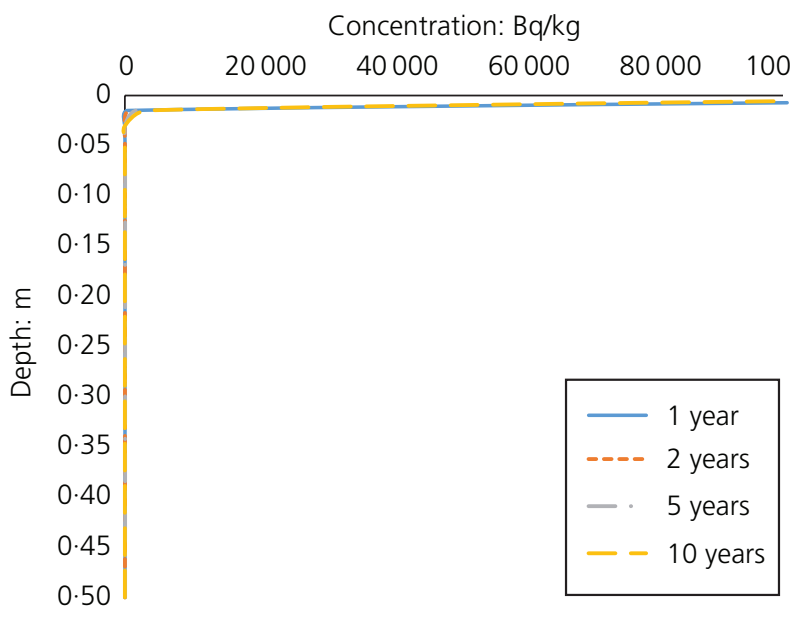

(c)

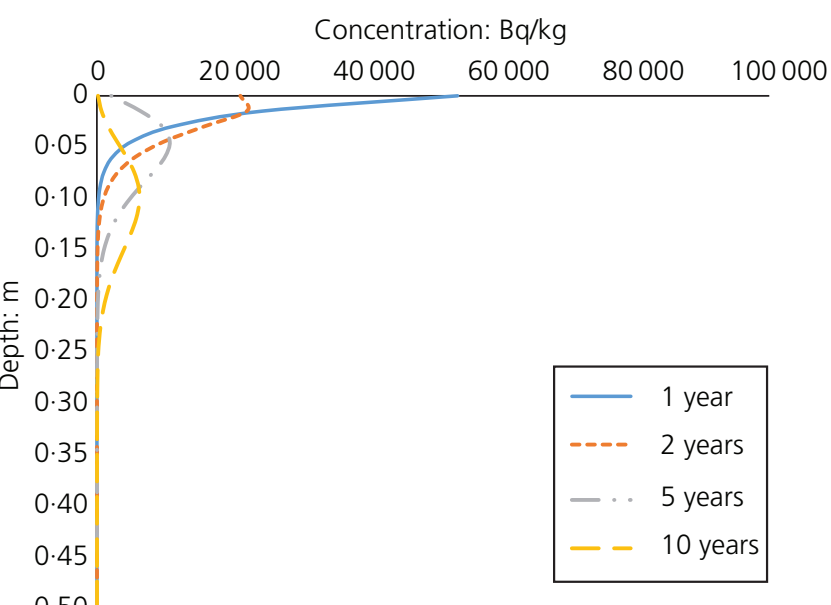

(b)

Concentration: $\mathrm{Bq} / \mathrm{kg}$

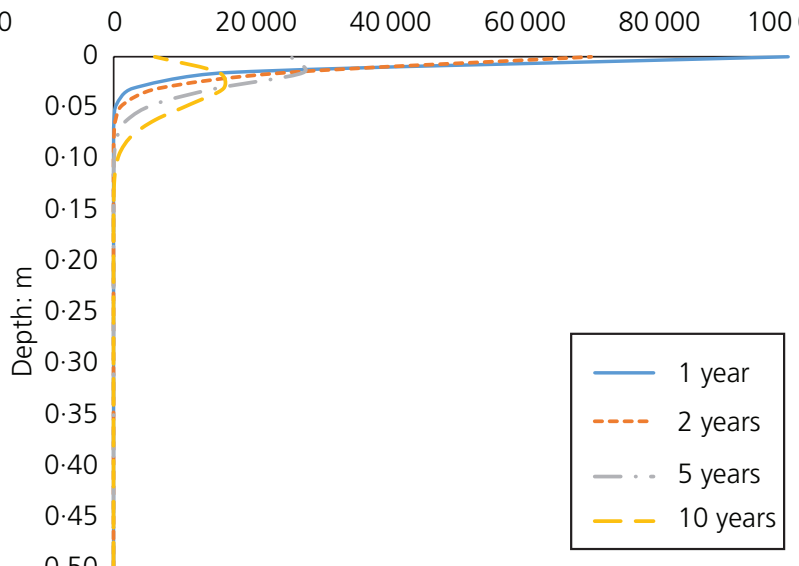

(d)

Figure 17. Concentration distribution at $0.7 \mathrm{~m}$ point in each layer: (a) in a silt layer; (b) in a sand layer; (c) in a clay layer; (d) in an alternate layer 
of the silt layer shown in Table 3 changed, the transition of the concentration at a point $0.7 \mathrm{~m}$ from the left-hand edge and $0.2 \mathrm{~m}$ deep was evaluated.

First, the hydraulic conductivity $k$ changed from $1.0 \times 10^{-4}$ to $1.0 \times 10^{-6} \mathrm{~m} / \mathrm{s}$ for each order, similar to that shown in Figure 18 . According to Figure 18, the transition of concentration by changing the hydraulic conductivity $k$ was $1.0 \times 10^{-4} \mathrm{~m} / \mathrm{s}$, and caesium-137 reached the point in earlier years. Then, the concentration gradually fell after the peak at 4 years of progress. When the hydraulic conductivity $k$ was $1.0 \times 10^{-5} \mathrm{~m} / \mathrm{s}$, the concentration increased after 5 years of progress. However, when the hydraulic conductivity $k$ was $1 \cdot 0 \times 10^{-6} \mathrm{~m} / \mathrm{s}$, the caesium-137 quantity did not reach the point even after 10 years of progress. Thus, significant changes in the transportation behaviour occurred based on the value of the hydraulic conductivity $k$.

Next, the concentration at the same point was observed when the value of the distribution coefficient $K_{\mathrm{d}}$ was changed from $4.0 \times$ $10^{-3}$ to $1.0 \times 10^{-2} \mathrm{~g} / \mathrm{m}^{3}$ based on the value of sand compared with

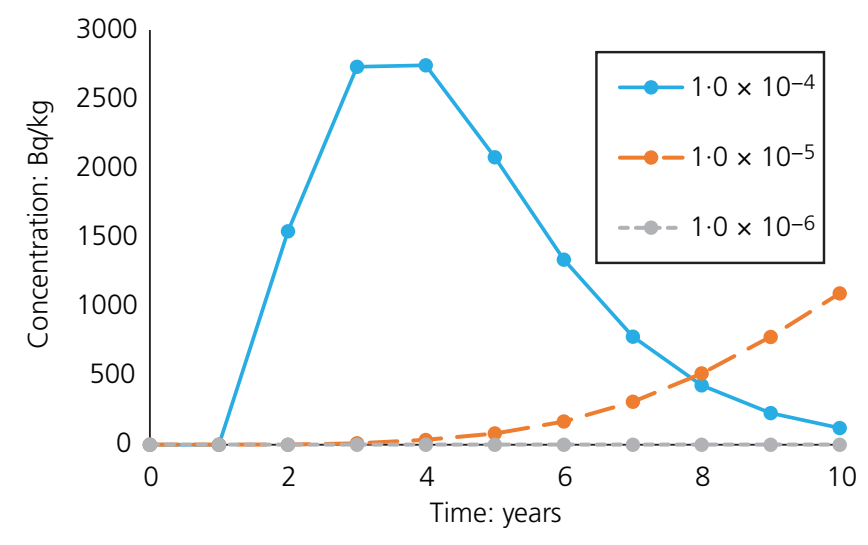

Figure 18. Concentration transition by changing the hydraulic conductivity $k$

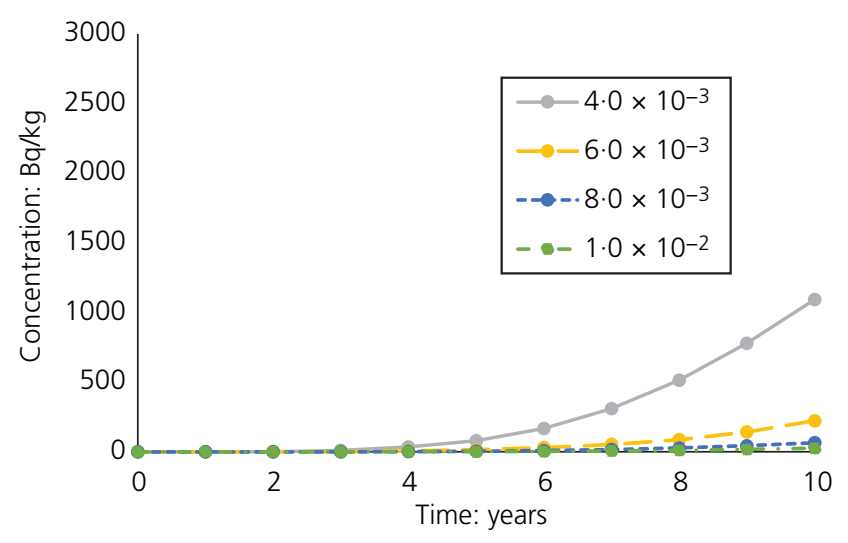

Figure 19. Concentration transition by changing the distribution coefficient $K_{\mathrm{d}}$ that of clay. Figure 19 shows this result. As Figure 19 indicates, the concentration after 10 years decreased as the distribution coefficient $K_{\mathrm{d}}$ reduced. However, there was no difference in the transportation case for the hydraulic conductivity $k$, and only the concentration ratio increased as the distribution coefficient $K_{\mathrm{d}}$ decreased. For the case where the concentration was the highest, the distribution coefficient $K_{\mathrm{d}}$ was $4.0 \times 10^{-3} \mathrm{~g} / \mathrm{m}^{3}$, and the increase was approximately $1000 \mathrm{~Bq} / \mathrm{kg}$. The influence of the distribution coefficient $K_{\mathrm{d}}$ was smaller than that of the hydraulic conductivity $k$.

Accordingly, it was confirmed that the advection term excels as an adsorption term. Therefore, the restraint of migration for a sand layer can be expected by making the hydraulic conductivity $k$ small, as in compaction. However, if the ground has a small hydraulic conductivity $k$, transportation behaviour by dispersion and diffusion needs to be considered.

\section{Influence of radioactive half-life}

The influence of radioactive half-life can be evaluated by comparing analysis results that considered radioactive half-life with those that did not. Figure 20 shows the concentration distribution for a sand layer at the $0.7 \mathrm{~m}$ point for these two cases. The results show that there is a difference of approximately $2000 \mathrm{~Bq} / \mathrm{kg}$ at the peak concentration. This difference is important for the environment since decay by radioactive half-life is not affected by an external force. The arrival depth was constant without considering radioactive half-life. Thus, a decay term will not affect transportation in the depth direction.

\section{Analytical evaluation for modern decontamination}

Dredging out a depth of approximately $5 \mathrm{~cm}$ as a decontamination method for the analysis section was effective for grounds with silt or clay layers. For ground with an alternate layer, it could be effective for decontaminating a clay layer as it was found that movement would not occur in this layer. Finally, if the ground was

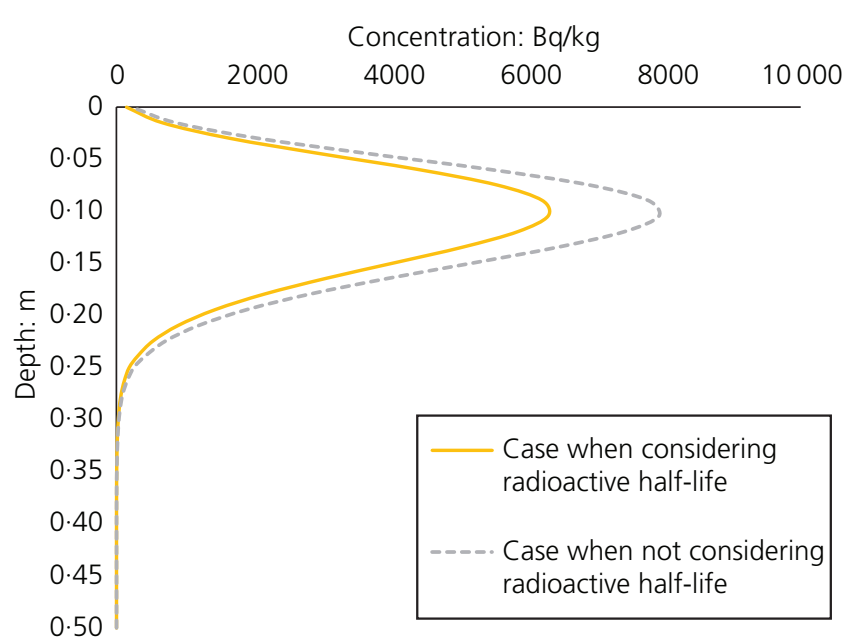

Figure 20. Difference in concentration distribution between cases where radioactive half-life was considered and not considered 
a sand layer, a portion of the caesium- 137 reached a $0 \cdot 1 \mathrm{~m}$ depth after 1 year. Thus, use of a sand layer is not effective in modern decontamination. Moreover, caesium-137 falling to the ground surface of section 2 after 5 years of progress may be a serious problem. Setting an impervious wall in the ground was proposed as a countermeasure for the sand layer. This is expected to be an effective suppression of transportation as radioactive substances stagnate around an impervious wall.

\section{Conclusions}

In this study, the transportation behaviour of radioactive substances in soils was evaluated by adding a term for the radioactive half-life to the advection-dispersion equation.

The conclusions are as follows.

- This analytical method can recreate the on-site situation through comparison of analysis results with measurement results.

- By carrying out tests for various soil properties, it was determined that the soil properties had an effect on transportation behaviour. Specifically, significant movement occurred in the sand layer. However, for the clay layer, the transportation behaviour was insignificant. Additionally, the radioactive substance remained in the clay layer when an alternate layer was used.

- The hydraulic conductivity $k$ had a significant effect on the transportation behaviour since the seepage and advectiondispersion analysis was in advection excellence mode.

- The influence of the radioactive half-life was evaluated by comparing it with the result obtained without considering the half-life. The arrival depth was constant with or without consideration of the radioactive half-life.

- Modern decontamination techniques were effective for the section under analysis for ground with a silt or a clay layer. However, these techniques were not effective for sand layers.

This analysis did not consider scattering and flowing out by weathering, nor absorption by plants. In other words, the analysis was not conducted under a non-steady state. If these factors were considered, the concentration of the radioactive substance would be likely to be small. A new factor would be required for the analysis equation. Finally, construction of radioactive waste disposal sites is becoming a social problem. Analytical safety evaluations of radioactive waste disposal sites should be conducted in conjunction with the results of this study.

\section{REFERENCES}

Bharat TV (2014) Analytical model for 1-D contaminant diffusion through clay barriers. Environmental Geotechnics 1(4): 210-221, http://dx.doi. org/10.1680/envgeo.13.00035.

Bossew P and Kirchner G (2004) Modelling the vertical distribution of radionuclides in soil. Part 1: the convection-dispersion equation revisited. Journal of Environmental Radioactivity 73(2): 127-150, http://dx.doi.org/10.1016/j.jenvrad.2003.08.006.
Desai YM, Eldho TI and Shah AH (2011) Finite Element Method with Applications in Engineering. Pearson Education India, Delhi, India, pp. 189-191.

Endo S, Kimura S, Takatsuji T et al. (2012) Measurement of soil contamination by radionuclides due to the Fukushima Dai-ichi Nuclear Power Plant accident and associated estimated cumulative external dose estimation. Journal of Environmental Radioactivity 111: 18-27.

Forestry Agency (2011) Forestry Agency, Tokyo, Japan. See http://www. rinya.maff.go.jp/j/press/kaihatu/pdf/150130-01.pdf (accessed 10/03/2016).

Gelhar LW and Axness CL (1983) Three-dimensional stochastic analysis of macrodispersion in aquifers. Water Resources Research 19(1): $161-180$.

Inoue $Y$ and Morisawa S (1976) Distribution coefficient Kd of radionuclide between sample soil and water. Journal of the Atomic Energy Society of Japan 18: 524-534.

Japanese Geotechnical Society (2002) Japanese Geotechnical Society, Tokyo, Japan. See http://www.jiban.or.jp/organi/bu/kijyunbu/kouji/ 200807/JGS 0311 080801.pdf (accessed 10/03/2016).

Kato $\mathrm{H}$, Onda $\mathrm{Y}$ and Teramage M (2012) Depth distribution of ${ }^{137} \mathrm{Cs}$, ${ }^{134} \mathrm{Cs}$, and ${ }^{131} \mathrm{I}$ in soil profile after Fukushima Dai-ichi Nuclear Power Plant accident. Journal of Environmental Radioactivity 111: 59-64, http://dx.doi.org/10.1016/j.jenvrad.2011.10.003.

Komamura M, Tsumura A, Yamaguchi N et al. (2006) Long-term monitoring and analysis of ${ }^{90} \mathrm{Sr}$ and ${ }^{137} \mathrm{Cs}$ concentrations in rice, wheat and soils in Japan from 1959 to 2000. Bulletin of the National Institute of Agro-environmental Sciences 24: 1-21.

Ministry of Education, Science and Culture (2011a) Ministry of Education Science and Culture, Tokyo, Japan. See http://radioactivity. nsr.go.jp/ja/contents/4000/3912/24/1305995_0512_1.pdf (accessed 10/03/2016).

Ministry of Education, Science and Culture (2011b) Ministry of Education Science and Culture, Tokyo, Japan. See http://radioactivity.nsr.go.jp/ja/ contents/6000/5233/24/5233_20120313_20120615_rev20130701.pdf (accessed 10/03/2016).

Ministry of Education, Science and Culture (2011c) Ministry of Education Science and Culture, Tokyo, Japan. See http://radioactivity.nsr.go.jp/ja/ contents/6000/5522/25/5600_201203131000_report2-1.pdf (accessed $10 / 03 / 2016)$

Ministry of Health, Labour and Welfare (2011) Ministry of Health, Labour and Welfare, Tokyo, Japan. See http://www.mhlw.go.jp/stf/ shingi/2r9852000001 tmph-att/2r9852000001u038.pdf (accessed 10/03/2016).

Ministry of the Environment (2011a) Ministry of the Environment, Tokyo, Japan. See http://www.env.go.jp/jishin/monitoring/ result gw110804.pdf (accessed 10/03/2016).

Ministry of the Environment (2011b) Ministry of the Environment, Tokyo, Japan. See http://www.env.go.jp/press/files/jp/20719.pdf (accessed 10/03/2016).

Ministry of the Environment (2011c) Ministry of the Environment, Tokyo, Japan. See https://www.env.go.jp/council/09water/y090-29/ mat06-3.pdf (accessed 10/03/2016).

Ministry of the Environment (2013) Decontamination Guidelines, 2nd edn. Ministry of the Environment, Tokyo, Japan. See http://josen. env.go.jp/en/framework/pdf/decontamination_guidelines_2nd.pdf (accessed 10/03/2016)

Mohammad V (2012a) Hydro-module determination for Vanaei village in Eslam Abad Gharb, Iran. ARPN Journal of Agricultural and Biological Science 7: 968-976.

Mohammad V (2012b) A comparison between horizontal and vertical drainage systems (include pipe drainage, open ditch drainage, and pumped wells) in anisotropic soils, Mohammad Valipour. IOSR Journal of Mechanical and Civil Engineering 4: 7-12.

Mohammad V (2013) Increasing irrigation efficiency by management strategies: cutback and surge irrigation. ARPN Journal of Agricultural and Biological Science 8: 35-43. 
Mohammad V (2015) Handbook of Environmental Engineering Problems. Omics Group eBooks, Hyderabad, India.

Morino Y, Ohara T and Nishizawa M (2011) Atmosphere behavior, deposition, and budget of radioactive materials from the Fukushima Daiichi nuclear power plant in March 2011. Geophysical Research Letters 38(7): 2011.

National Institute for Environmental Studies (2012) National Institute for Environmental Studies, Tsukuba, Japan. See http://kinki.env.go.jp/ to_2012/data/0606ab.pdf (accessed 10/03/2016).

National Institute of Advanced Industrial and Technology (2012) National Institute of Advanced Industrial and Technology, Tokyo, Japan. See http://www.aist.go.jp/aist_j/new_research/2012/nr20120905/ nr20120905.html (accessed 10/03/2016).

Nguyen TS, Li Z, Garitte B and Barnichon JD (2017) Modelling a heater experiment for radioactive waste disposal. Environmental Geotechnics, https://doi.org/10.1680/jenge.15.00060.

Ochiai T, Takeda S and Kimura H (2009) Database on Soil-to-Plant Transfer Factors for the Safety Assessment of Geological Disposal. Japan Atomic Energy Agency, Ibaraki, Japan.
Radioactive Waste Management Center (1990) Radionuclides Distribution Coefficient of Soil to Soil-Solution. Radioactive Waste Management Center, Japan.

Saito K, Aoki K and Tanimura I (2012) Radioactive Matter in Soil. Confirmation of the Depth Distribution of Quality. See http://fukushima. jaea.go.jp/initiatives/cat01/pdf05/02-03.pdf (accessed 10/03/2016).

Shiozawa S, Tanoi K, Nemoto K et al. (2011) Vertical concentration profiles of radioactive caesium and convective velocity in soil in a paddy field in Fukushima. Radioisotopes 60(8): 323-328.

Tsukada H, Shibata H and Sugiyama H (1998) Transfer of radioactive caesium and stable caesium from substrata to mushrooms in a pine forest in Rokkasho-mura, Aomori, Japan. Journal of Environmental Radioactivity 39: 149-160.

UNSCEAR (UN Scientific Committee on the Effects of Atomic Radiation) (1988) UNSCEAR, Austria, Vienna, UNSCEAR 1988 Report, p. 24

Vardon PJ and Heimovaara TJ (2015) Waste barriers in environmental geotechnics. Environmental Geotechnics, http://dx.doi.org/10.1680/ envgeo.13.00100.

\section{How can you contribute?}

To discuss this paper, please submit up to 500 words to the editor at journals@ice.org.uk. Your contribution will be forwarded to the author(s) for a reply and, if considered appropriate by the editorial board, it will be published as a discussion in a future issue of the journal. 